\title{
The density matrix via few dominant observables: the quantum interference in the isotope effect for atto-pumped $\mathrm{N}_{2}$
}

\author{
K. Komarova, ${ }^{1, a)}$ F. Remacle, ${ }^{1,2}$ and R. D. Levine ${ }^{1,3}$ \\ ${ }^{1}$ The Fritz Haber Center for Molecular Dynamics and Institute of Chemistry, The Hebrew University of \\ Jerusalem, Jerusalem 91904, Israel \\ ${ }^{2}$ Theoretical Physical Chemistry, UR MolSys B6c, University of Liège, B4000 Liège, Belgium \\ ${ }^{3}$ Department of Chemistry and Biochemistry and Department of Molecular and Medical Pharmacology, \\ David Geffen School of Medicine, University of California, Los Angeles, CA 90095, USA
}

\begin{abstract}
Atto- and sub-femto-photochemistry enables preparation of molecules in a coherent superposition of several electronic states. Recently [J. Ajay et al, PNAS 2018] we examined an effect of the nuclear mass during the non-adiabatic transfer between strongly coupled Rydberg and valence electronic states in $\mathrm{N}_{2}$ excited by an ultrafast pulse. Here we develop and analyze an algebraic description for the density matrix and its logarithm, the surprisal, in such a superposition of states with a focus on the essentially quantum effect of mass. This allows the identification of a few observables that accurately characterize the density matrix of the system with several coupled electron-nuclear states. We compact the time evolution in terms of the time dependent coefficients of these observables.

Using the few observables we derive an analytical expression for the time-dependent surprisal. This provides a mass-dependent phase-factor only in the observables off-diagonal in the electronic index. The isotope effect is shown to be explicitly driven by the shift in equilibrium position of the valence state potential. It is analytically given as a time-dependent phase factor describing the interference in the overlap of the two wave packets on the coupled electronic states. This phase factorizes as a product of classical and quantal contributions.
\end{abstract}

\footnotetext{
a) Author to whom correspondence should be addressed: ksenia.komarova@mail.huji.ac.il
} 


\section{INTRODUCTION}

Interference amongst discrete states is an inherently quantal effect. Over the years experimental spectroscopy could coherently excite states separated from one another by an ever increasing energy gap. Earliest was NMR when the gap between states of nuclear spins up and down in a magnetic field was in the microwave region. The corresponding period was in the time scale of molecular processes in condensed phases. More recently came 'femtochemistry ${ }^{11}$ where localized wave packets of the motion of the atoms could be prepared as coherent superpositions of stationary vibrational states. Currently, with attosecond laser pulses, interference can be observed between different electronic states. In molecules, such an interference between different electronic states can result in an unprecedented fast migration of charge across the molecular backbone. ${ }^{2}$ This process that is dependent on the coherence between the wave packets moving on different electronic states and is distinct from electronic state reorganization induced by the motion of the nuclei. We here adapt the dependence of the motion of the nuclei on their mass to untangle these two contributions to the charge reorganization. The task is made simpler by working in a diabatic basis of electronic states. Potential energy curves can therefore cross and the coupling between them is through a mass-independent potential energy term.

The curve crossing problem is extensively discussed ${ }^{3 / 4}$ since Landau and Zener.5 The new aspect in this problem is the special way of preparing molecule in excited electronic states. The molecule is pumped by a coherently broad in energy ultrafast pulse hence our initial conditions for the non-adiabatic transfer are such that both of the electronic states are already initially populated. It is the interference between the vibrational wave packets on these two states that is at the center of our attention. In a density matrix language, we highlight the evaluation of the off-diagonal elements of the density matrix. As we shall see the mass dependent phase difference between the wave packets on the two electronic states can make the transfer go in either direction. The formal connection to the isotope effect

in the non-adiabatic transfer ${ }^{[6}$ is that the rate of change of the population between the two states is determined by their coherence..$^{7}$

It was shown by Heller ${ }^{8}$ that a motion of the coherent state already even on a single harmonic potential can generate a phase factor. This phase cancels out for the diagonal 
elements of a density matrix and it is only through the coherence of two different electronic states that this phase can give rise to observable effects. In this paper we formulate the dynamics in Liouville space so that we can trace the origin of the mass-dependent phase. We do so by expanding the density and its logarithm, the surprisal, in a small set of observables. In particular we need to use observables that are sufficient to describe both the electronic and the nuclear motions. The mass-dependent phase-factor is present only in the observables off-diagonal in the electronic index. For three electronic states this means three observables. The analytical derivation allows the factorization of the role of the mass in the dynamics into a classical and a quantal effect. By classical we mean that a heavier mass moves more slowly. The quantal effect is described by the mass-dependent phase-factor.

Our aim is to demonstrate the role of the mass on the inter electronic coherence via an algebraic route to the density matrix. The primary benefit of an algebraic approach is that it allows identifying a few dominant operators (= constraints) that accurately characterize the density matrix. This is not only computationally useful but also provides physical insight. We here use the isotope effect to illustrate both these aspects.

To describe the dynamics of coupled electronic states we implement an algebraic formulation of quantum dynamics that is motivated by an information-theoretic approach. ${ }^{911}$ It is a predictive, dynamical, version of the procedure known as surprisal analysis ${ }^{12[13}$ In essence the dynamical approach seeks to identify such operators, that we call 'constraints', whose mean values are sufficient to provide an exact or realistically approximate description of the dynamics. We use the term 'sufficient' in its technical meaning in statistics 14 The distribution of interest depends only on the mean value of (one or more) sufficient statistics. The formalism of maximal entropy ${ }^{15 \mid 16}$ identifies a sufficient statistic as a mean value which, when the distribution is constrained to the given value, lowers the entropy of the distribution. A distribution of maximal entropy that is consistent with the given mean value of a sufficient statistic can be shown to be of the exponential type. Important details need to be addressed when the distribution is quantum mechanical ${ }^{17 \mid 18}$ but the final conclusion remains. In the dynamical, predictive approach the sufficient statistics are identified not by an analysis of data but by using the change in time induced in a given state by the Hamiltonian of the system. In the quantum variant of the predictive approach one finds an expression for the density matrix as an exponential function of not necessarily commuting quantum-mechanical oper- 
ators. The mean values of these operators serve as constraints in lowering the information entropy of the density matrix. The final result is $\hat{\rho}=\exp \left(-\sum_{k} \lambda_{k}(t) \hat{A}_{k}\right){ }^{9111}$ with the time dependence carried by the Lagrange multipliers $\lambda_{k}(t)$. Below we discuss computing the time dependence of the Lagrange multipliers for the quantum multi electronic state problem. This allows a compact representation of the surprisal and thereby of the density matrix. Specifically in our example we will need far fewer than $10 \%$ of the number of operators needed to fully characterize the quantal system.

Detailed distributions of final electronic states have been measured for a variety of very exothermic chemical reactions where energy is plentiful. ${ }^{19}$ Interestingly, often the observed distributions are essentially statistical meaning that they are of maximal entropy without the need to impose any dynamical constraints. One can speculate whether this is due to the multitude of curve crossings that is typical of such triatomic or larger systems. ${ }^{22}$ Here however we discuss a single curve crossing and the constraints that are needed to describe the time evolving dynamics.

To focus on the essence we determine the constraints for a 'toy model' of a curve crossing. It is a model that has been validated against exact dynamics ${ }^{6}$ and has been shown to reproduce the role of the mass and other key features. The crossing is between excited (diabatic) Rydberg and valence electronic states of $\mathrm{N}_{2}$. In the toy model the two potentials are taken to be harmonic with the valence excited state having a larger equilibrium distance than the Rydberg state, see Fig. 1. The diabatic coupling of the two states is localised in the Franck-Condon region and set to be a linear fit to the coupling identified by Spelsberg and Meyer. ${ }^{23}$ An initial state that is a coherent superposition of these two, b and c, electronic states of $\mathrm{N}_{2}$ is prepared by an ultrafast pulse. We seek to identify the sufficient statistics by algebraic means and analyze its mass-dependence.

In this paper we examine the isotope effect in the distribution of electronic and vibrational states. Already in the very first applications of surprisal analysis $\frac{13 \mid 24}{\sqrt{2}}$ the role of the isotope in the distribution of vibrational states in electronically adiabatic processes received central attention. The early example was $\mathrm{Cl}+\mathrm{HI}$ vs $\mathrm{Cl}+\mathrm{DI} 1324$ The clear and simple answer was that the distribution of products vibrational energy is essentially independent of mass. So the distribution of vibrational energy in $\mathrm{HCl}$ is the same as that of $\mathrm{DCl}$ when plotted vs. the vibrational energy. An example for the four reactions $\mathrm{F}+\mathrm{H}_{2}, \mathrm{~F}+\mathrm{D}_{2}, \mathrm{~F}+\mathrm{HD}$ and $\mathrm{F}+\mathrm{DH}$ 


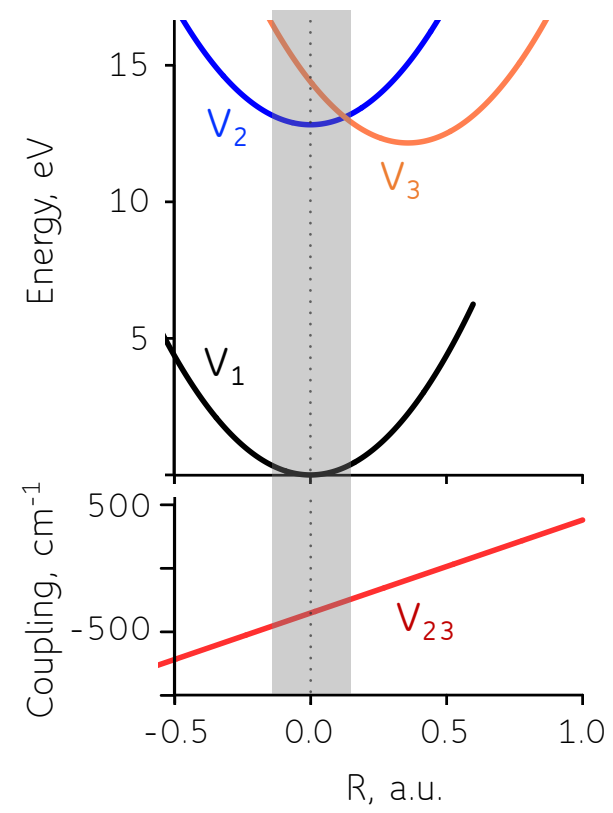

FIG. 1. Diabatic potentials for the ground $\left(V_{1}\right.$, black) and two excited electronic states $\left(V_{2}\right.$ and $V_{3}$ in blue and orange, respectively). The Franck-Condon region is shown as a gray shaded area around the equilibrium position $R_{e q}=0$ of the ground state. The diabatic coupling between the excited states $\left(V_{23}\right.$, in $\left.\mathrm{cm}^{-1}\right)$ is shown in red. The coupling is delocalized but as emphasized by Landau and by Zener ${ }^{[5}$ the effective coupling is localized about the diabatic curve crossing region.

is figure 4.26 of Ref. 25. Soon after, the same behavior was found for the distribution of rotational energy. A relation between the distribution of products vibrational states and the overlap between products vibrational wave functions with the wave function of the reactants was indeed proposed early on $\sqrt[2627]{27}$ The difference between these experiments and the present system is that here we deal with electronic non-adiabatic transitions resulting upon an attosecond excitation. The view of the dynamics in a time-domain is therefore more suitable than the picture of the dynamics in an energy domain.

The paper is organized in three sections. Sec. II provides details of the computation where the motion of the surprisal is discussed in section II.B. The concept of the dominant constraints, the major contributors to the surprisal is outlined in section II.C. The key computational results and their interpretation are discussed in section III. In particular, the isotope effect computed both in an exact dynamical computation and by retaining only the dominant constraints is presented. An analytical interpretation of the role of the mass 
is provided in section IV. The (analytical) mass dependence of the phase in the Lagrange multipliers of the constraints off-diagonal in the electronic state is the main result.

\section{METHODS AND COMPUTATIONAL DETAILS}

\section{A. Model potentials, coupling and parameters of the laser field}

We use model potentials similar to the one defined in Ref. 6 in the toy-model. This toymodel aims to reproduce the strong isotope effect that is computed ${ }^{6}$ in the case of realistic potentials and couplings in ${ }^{1} \Pi_{u}$ excited states of $\mathrm{N}_{2} \cdot{ }^{23}$ It allows an algebraic description of the dynamics and enables to get the origin of the effect of mass on the non-adiabatic transfer.

In the toy-model the diabatic potentials of the electronic states involved in the dynamics are taken to be harmonic with the same force constant but different position of equilibrium internuclear distance. In the absence of coupling between the diabatic states a Gaussian wave packet, a coherent state, will remain Gaussian. The ground electronic state $V_{1}$ and the first excited (Rydberg) state $V_{2}$ are assumed to have the same equilibrium distance, while the potential for the second excited state $V_{3}$, a prototype of the valence state, has significant shift in its equilibrium position, $\delta R=0.36$ a.u., see Fig. 1 and Table $\mathrm{S} 1$ in section $\mathrm{S} 1$ of the supplementary material. In this toy model we are able to identify a small set of dominant constraints and to determine a dimensionless parameter for the mass dependence.

The diabatic coupling between the two excited states is localized in the Franck Condon region. At the onset of the dynamics the wave packet on the shifted potential moves out of the coupling region. The other wave packet, the one on the Rydberg like state, stays behind and can spawn amplitude on the shifted potential in a region that is essentially empty. This is an only weakly mass dependent unistate transfer. ${ }^{6}$ The strongly mass dependent bistate transfer occurs later when the wave packet that moved out returns to the coupling region. In the toy model, to reduce the unistate transfer the coupling is acting only upon the recurrence of the $V_{3}$ wave packet in the coupling region. We also use a coupling that is twice weaker than the realistic diabatic coupling in $\mathrm{N}_{2}$ as given in Ref. 23. The trends in the mass dependence remain similar to the full exact computations as reported in Ref. 6 . 
The total Hamiltonian is written as follows:

$$
\begin{aligned}
\hat{H}= & |1\rangle \hat{h}_{1}\langle 1|+| 2\rangle \hat{h}_{2}\langle 2|+| 3\rangle \hat{h}_{3}\langle 3|+\left(|2\rangle \hat{V}_{23}\langle 3|+| 3\rangle \hat{V}_{23}\langle 2|\right) \\
& -\mu_{12} E(t)(|1\rangle \hat{\mathbb{I}}\langle 2|+| 2\rangle \hat{\mathbb{I}}\langle 1|)-\mu_{13} E(t)(|1\rangle \hat{\mathbb{I}}\langle 3|+| 3\rangle \hat{\mathbb{I}}\langle 1|)
\end{aligned}
$$

The operator $|i\rangle \hat{\mathbb{I}}\langle i|$ is a projector on a basis of the ground $(i=1)$ and the two excited $(i=2,3)$ electronic states involved in the dynamics, with $\hat{\mathbb{I}}$ being the identity operator in the supspace of vibrations. Operators $\hat{h}_{i}$ are harmonic Hamiltonians of the three uncoupled electronic states, $\hat{h}_{3}$ has a shifted equilibrium position. A more detailed form of the Hamiltonian is given in section S1 of the supplementary material. The coupling of the diabatic states is fitted to a linear form in the nuclear displacement, $\hat{V}_{23}=c_{0}+c_{1} \cdot \hat{R}\left(c_{0}=-0.0016\right.$ $E_{h}$ and $\left.c_{1}=0.0033 E_{h} \cdot a_{0}^{-1}\right)$. The transition dipole moments from the ground to two excited states, $\mu_{12}$ and $\mu_{13}$, are taken to be constant in nuclear displacement and correspond to a realistic transition dipoles for the ground to Rydberg and ground to valence state excitations defined at the Franck-Condon region $\left(\mu_{12}=-0.27\right.$ a.u. and $\mu_{13}=-0.64$ a.u.). ${ }^{23}$ Atomic units are used throughout $(\hbar=1)$.

The interaction with the XUV-pulse is described by taking into account the explicit time profile of the laser field:

$$
\vec{E}(t)=\vec{E}_{p} \exp \left(-\left(t-t_{p}\right)^{2} / 2 \sigma_{p}^{2}\right)\left[\cos \left(\omega_{p} t\right)-\frac{\left(t-t_{p}\right)}{\omega_{p} \sigma_{p}^{2}} \sin \left(\omega_{p} t\right)\right]
$$

where $t_{p}$ and $\sigma_{p}$ are mean value and the variance of the envelope of the pulse. These parameters of the envelope correspond to less than 1 fs duration of the pulse centered about 2.4 fs. The frequency $\omega_{p}$ is 0.5 a.u. $\left(109.7 \cdot 10^{3} \mathrm{~cm}^{-1}\right)$ which is high enough to excite both $V_{2}$ and $V_{3}$ electronic states. The polarization of the laser field, $\vec{E}_{p}$ is taken to be perpendicular to the interatomic bond.

\section{B. Time-evolution of the surprisal and its transformation to the density matrix}

In order to identify the sufficient statistics from the results of a numerical quantum dynamics on multiple electronic states we need to compute the time-evolution of the surprisal, the logarithm of the density operator $\hat{I}=-\ln \hat{\rho}$. Here we propagate the surprisal in a finite basis representation that achieves numerical convergence. 11

The choice of the basis states is dictated on the one hand by the fact that the initial wave packets pumped by an ultrafast excitation are localized in the Franck Condon region. On 
the other hand the highest excited state has a potential with a shifted equilibrium distance. So a coherent superposition of stationary vibrational states is a good representation for a possibly large number of the excited vibrational states. We use throughout this paper a finite basis of $N=5$ coherent vibrational states, $\frac{28}{28}\left\{\left|i, \alpha_{k}\right\rangle\right\}$, for each electronic state $i$. This rather small set of coherent states is sufficient to represent the dynamics in particular because on the third state we use functions that are moving in time.

At time $t=0$ the basis functions are real functions of the nuclear coordinate $R$, displacement from equilibrium position of the ground electronic state, and differ only by their mean position, $\langle R\rangle_{\alpha_{k}}=R_{k}$ :

$$
\left|\alpha_{k}(0)\right\rangle=\left(\frac{m \omega}{\pi}\right)^{\frac{1}{4}} \exp \left(-\frac{m \omega}{2}\left(R-R_{k}\right)^{2}\right)
$$

Here $\omega$ is the frequency of the toy-model potentials and $m$ is the reduced mass, both are defined for each isotopomer studied. The mean values $R_{k}$ are set to span the distance [-2, 2] a.u. so that for $N=5$ these basis functions are effectively not overlapping.

There is no nuclear dynamics on the ground and first excited potentials so their basis functions are not changing with time. The potential $V_{3}$ is shifted in equilibrium position compare to the other two electronic states, hence the nuclear basis functions for this state are taken to be time-dependent. The time-dependence of the basis functions on $V_{3}$ potential is defined using a time-dependent shift operator $\hat{D}: \underline{28}$

$$
\begin{aligned}
& \left|3, \alpha_{k}(t)\right\rangle=\hat{D}(\eta(t))\left|3, \alpha_{k}(0)\right\rangle=e^{i \alpha_{k} \operatorname{Im}\{\eta\}}\left|\eta(t)+\alpha_{k}(0)\right\rangle \\
& \hat{D}(\eta(t))=\exp \left(\eta(t) \hat{a}^{\dagger}-\eta(t)^{*} \hat{a}\right) \\
& \eta(t)=\xi\left(1-e^{-i \omega t}\right), \quad \xi=\sqrt{m \omega / 2} \cdot R_{e q}
\end{aligned}
$$

The time-dependent shift $\eta(t)$ is determined from the analytical solution of the evolution of the surprisal after the laser field is over, see section S1 of the supplementary material for the details. The shift of the center $R_{k}$ and mean momentum is the same for all the basis functions on the electronic state 3. The mass dependence of the shift is governed by the dimensionless parameter $\xi$ that is linear in the shift of the valence potential equilibrium distance, $R_{e q}$, times $\sqrt{m \omega}$. As will be argued later the quantum effect on the coherence during the transfer is quadratic in $\xi$. The classical effect is determined by the above $\eta(t)$ that is linear in $\xi$. This means that the quantum effect is more sensitive to the details of the potentials. 
The rate of change of the surprisal in time is defined by its commutation relation with the Hamiltonian, Eq. (1):

$$
\frac{\mathrm{d} \hat{I}}{\mathrm{~d} t}=-i[\hat{H}, \hat{I}]
$$

The basis functions depend on time therefore the time-derivatives of the matrix elements of the surprisal involve also the derivatives of the basis functions wrt time:

$$
\begin{aligned}
& \frac{\mathrm{d}}{\mathrm{d} t}\left\langle\alpha_{m}(t), i|\hat{I}(t)| j, \alpha_{k}(t)\right\rangle=\left\langle\alpha_{m}(t)\left|\frac{\mathrm{d} \hat{I}^{i j}}{\mathrm{~d} t}\right| \alpha_{k}(t)\right\rangle \\
& +\left\langle\frac{\mathrm{d} \alpha_{m}(t)}{\mathrm{d} t}\left|\hat{I}^{i j}(t)\right| \alpha_{k}(t)\right\rangle+\left\langle\alpha_{m}(t)\left|\hat{I}^{i j}(t)\right| \frac{\mathrm{d} \alpha_{k}(t)}{\mathrm{d} t}\right\rangle
\end{aligned}
$$

see section $\mathrm{S} 2$ of the supplementary material for more details on the propagation routine via a Runge-Kutta 4th order propagator.

Populations and coherences between the electronic states are tracked throughout the dynamics by diagonalization of the surprisal matrix and subsequent transformation to the density using the spectral representation of the surprisal:

$$
\begin{aligned}
& \hat{I}(t)=\sum_{s=0}^{3 N} \mu_{s}|s(t)\rangle\langle s(t)| \\
& \hat{\rho}(t)=\exp (-\hat{I}(t))=\sum_{s=0}^{3 N} \exp \left(-\mu_{s}\right)|s(t)\rangle\langle s(t)|
\end{aligned}
$$

Here $\left\{\mu_{s},\langle s(t)|\right\}$ - is a pair of eigenvalue and eigenvector of the surprisal given at a time $t$. $N=5$ is the number of basis functions per electronic state. Although at time $t=0$ the basis functions are effectively not overlapping, as the dynamics unfolds, Eq. (4), the off-diagonal matrix elements of the overlap matrix can increase. To account for the non-orthogonality of our vibrational basis functions we transform the matrix of the surprisal to the basis of eigenstates of the overlap matrix at each time step of the dynamics.

It is quite often of interest to consider the dynamics for an initial state that is a pure state. Under a unitary time evolution the density matrix will remain a pure state. This means that in Eq. (7) there is one eigenstate that dominates. It is the overwhelmingly larger eigenvalue of the density matrix or the smallest eigenvalue of the surprisal. That it requires a limiting operation is the mathematical statement of the physical restriction on reaching a pure state.

Initial state is the state of maximal entropy, a thermal state $\hat{\rho}(0)=Z^{-1} \exp \left\{-\beta \hat{H}_{0}\right\}$. Its 
surprisal is given by:

$$
\begin{aligned}
& \hat{I}(0)=-\ln \hat{\rho}(0)=\ln Z+\beta \hat{H}_{0}=V_{1}|1\rangle \hat{\mathbb{I}}\left\langle 1\left|+V_{2}\right| 2\right\rangle \hat{\mathbb{I}}\left\langle 2\left|+V_{3}\right| 3\right\rangle \hat{\mathbb{I}}\langle 3| \\
& +\beta \omega|1\rangle \hat{a}^{\dagger} \hat{a}\langle 1|+\beta \omega| 2\rangle \hat{a}^{\dagger} \hat{a}\langle 2|+\beta \omega| 3\rangle \hat{a}^{\dagger} \hat{a}\langle 3| \\
& V_{1}=\ln Z+\frac{1}{2} \beta \omega \quad V_{2}=\ln Z+\frac{1}{2} \beta \omega+\beta V_{2}^{(0)} \quad V_{3}=\ln Z+\frac{1}{2} \beta \omega+\beta V_{3}^{(0)}
\end{aligned}
$$

here $\hat{a}$ and $\hat{a}^{\dagger}$ are creation and annihilation operators ${ }^{28}$ and $V_{i}^{(0)}=V_{i}\left(R_{e q}\right)$ is the potential energy at the equilibrium distance for the electronic state $i$. The partition function is given by: $Z=\sum_{s=0}^{3 N} \exp \left(-\beta \epsilon_{s}\right)$, where $\left\{\epsilon_{s}\right\}$ are the eigenvalues of the harmonic Hamiltonian $\hat{H}_{0}$. The matrix elements of the operators involved in $\hat{I}(0)$ in the basis of coherent states are analytical:

$$
\begin{aligned}
& \left\langle\alpha_{k}(0), i \mid i\right\rangle \hat{\mathbb{I}}\left\langle i \mid j, \alpha_{m}(0)\right\rangle=\left\langle\alpha_{k}(0) \mid \alpha_{m}(0)\right\rangle \delta_{i j} \\
& \left\langle\alpha_{k}(0), i \mid i\right\rangle \hat{a}^{\dagger} \hat{a}\left\langle i \mid j, \alpha_{m}(0)\right\rangle=\alpha_{k}^{*}(0) \alpha_{m}(0)\left\langle\alpha_{k}(0) \mid \alpha_{m}(0)\right\rangle \delta_{i j} \\
& \left\langle\alpha_{k}(0) \mid \alpha_{m}(0)\right\rangle=e^{-\left|\alpha_{k}(0)\right|^{2} / 2} e^{-\left|\alpha_{m}(0)\right|^{2} / 2} e^{\alpha_{k}^{*}(0) \alpha_{m}(0)}
\end{aligned}
$$

See more details about matrix elements of coherent states in Ref. 28.

The inverse temperature $\beta=(k T)^{-1}$ is set to large enough value to approach the numerical limit of a pure state, the lowest vibrational state of the ground electronic state of the Hamiltonian $\hat{H}_{0}, \beta \cdot \omega=40$. This allows us to use a wave function propagation on a $\operatorname{grid}^{29 \mid 30}$ as a numerical benchmark for comparison with the surprisal dynamics. The initial state on a grid is defined via the only one eigenvector of the density that has a non-zero eigenvalue, $|s(0)\rangle$ Eq. (7). More details about the grid propagation are given in section S3 of the supplementary material.

\section{Determining a set of dominant constraints}

For discussing the density matrix and the surprisal of a quantum system we use observables, Hermitian operators that act as constraints. It is clear that the initial state of the surprisal, defined by Eq. (8), is a linear combination of a set of six Hermitian operators. Therefore its propagation in time can be written using the unitary time-evolution operator, $\hat{U}=$ $\exp (-i \hat{H} \cdot t)$, governed by the Hamiltonian Eq. (1):

$$
\hat{I}(t)=\hat{U}(t) \hat{I}(0) \hat{U}^{\dagger}(t)=\sum_{k}^{M_{0}} \lambda_{k} \hat{U}(t) \hat{A}_{k}(0) \hat{U}^{\dagger}(t)
$$


where $\left\{\hat{A}_{k}(0), \lambda_{k}\right\}$ is the set of $M_{0}$ operators, here $M_{0}=6$, with their coefficients, the Lagrange multipliers. The time-evolution of the initial state is thereby driven by the evolution of the initial set of constraints. Our main goal is to rewrite this expression in the following form:

$$
\hat{I}(t) \approx \sum_{k}^{M} \lambda_{k}(t) \hat{A}_{k}
$$

where the time-dependence is fully compacted into the vector of $M$ Lagrange multipliers. Here the constraints $\hat{A}_{k}$ are not changing with time. Their mean values still can be timedependent, governed by the time-dependence of the density, $\hat{\rho}=\exp \left(-\sum_{k}^{M} \lambda_{k}(t) \hat{A}_{k}\right)$. The number of operators that affect the dynamics can be different from the initial set of constraints, $M \geq M_{0}$. The equality occurs for the dynamics that is closed upon commutation with the Hamiltonian for all the initial constraints:

$$
\left[\hat{A}_{k}, \hat{H}\right]=\sum_{s}^{M_{0}} g_{k s} \hat{A}_{s}
$$

In this closed case Eq. (11) becomes exact. $\stackrel{911}{9}$ If the evolution of the operators in Eq. (10) is not closed, the set of constraints in Eq. (11) can be significantly larger (in principle, infinite) than that needed to describe the initial state and the problem reduces to the search for the minimal set of dominant observables. In our example $M_{0}=6$ involves only observables diagonal in the electronic index. The interaction with the field must raise this number to 9 to include off diagonal elements. In practice we find that $M=9$ is sufficient. This finite set of 9 operators are the dominant constraints.

It may be of interest to discuss what is the numerically exact number of constraints $M$. For the evolution of the matrix elements of the surprisal we find that it takes 5 basis functions per electronic state. So for the desired numerical accuracy the Hilbert space is 15 dimensional. The matrices in the Liouville space are therefore 15 by 15 . To represent all possible such Hermitian matrices one needs 15 by 15 independent matrices as a basis. The result that $M=9$ basis matrices are numerically enough is then a significant compaction of the dynamics.

The dynamics driven by the Hamiltonian, Eq. (1), leads to a not closed set of constraints. A large amplitude motion of the wave packet on the shifted potential gives rise to an exponential shift operator in the off-diagonal in the electronic index matrix elements 
of the surprisal, see details in section S1 of the supplementary material. Yet it is often the case that using a finite and small set of operators already offer a good approximation for the surprisal. ${ }^{[1]}$ In the absence of coupling between the diabatic states closure is enabled by using a time-dependent basis of coherent vibrational states on the shifted electronic state, Eq. (4). In the presence of coupling it is only closure of dominant constraints. A detailed derivation in section S1 of the supplementary material shows that the matrix elements of the surprisal in the time-dependent basis can be written as a linear superposition of the matrix elements of the initial set of constraints but in the basis of non-shifted coherent states $\left|\alpha_{n}(0)\right\rangle$, for example:

$$
I_{m n}^{23}(t)=\left\langle\alpha_{m}(0), 2|\hat{I}(t)| 3, \alpha_{n}(t)\right\rangle=\sum_{k}^{M_{0}} \lambda_{k}^{23}(t)\left\langle\alpha_{m}(0)\left|\hat{A}_{k}\right| \alpha_{n}(0)\right\rangle
$$

In what follows we use $\left\langle\alpha_{m}\left|\hat{I}^{23}\right| \alpha_{n}(t)\right\rangle=\left\langle\alpha_{m}(0), 2|\hat{I}(t)| 3, \alpha_{n}(t)\right\rangle$ shorthand notation for clarity.

In order to identify this dominant set of constraints we compute the time-dependent Lagrange multipliers from the exact surprisal, propagated in the finite basis, section II.B. The minimal set of constraints is listed in Table I.

TABLE I. Dominant set of 9 operators and their conjugate Lagrange multipliers: (a) operators diagonal in the electronic index; (b) operators off-diagonal in the electronic index. The operators off-diagonal in the electronic index are listed without their hermitian conjugates as their Lagrange multipliers are not independent, $i \neq j:\left(\lambda_{k}^{i j}\right)^{*}=\lambda_{k}^{j i}$, to keep the surprisal Hermitian.

\begin{tabular}{|c|c|c|c|}
\hline (a) & $|1\rangle\langle 1|$ & $|2\rangle\langle 2|$ & $|3\rangle\langle 3|$ \\
\hline$\hat{\mathbb{I}}$ & $|1\rangle \hat{\mathbb{I}}\langle 1|, \lambda_{\mathbb{I}}^{11}$ & $|2\rangle \hat{\mathbb{I}}\langle 2|, \lambda_{\mathbb{I}}^{22}$ & $|3\rangle \hat{\mathbb{I}}\langle 3|, \lambda_{\mathbb{I}}^{33}$ \\
\hline$\hat{a}^{\dagger} \hat{a}$ & $|1\rangle \hat{a}^{\dagger} \hat{a}\langle 1|, \lambda_{a^{\dagger} a}^{11}$ & $|2\rangle \hat{a}^{\dagger} \hat{a}\langle 2|, \lambda_{a^{\dagger} a}^{22}$ & $|3\rangle \hat{a}^{\dagger} \hat{a}\langle 3|, \lambda_{a^{\dagger} a}^{33}$ \\
\hline (b) & $|1\rangle\langle 2|$ & $|1\rangle\langle 3|$ & $|2\rangle\langle 3|$ \\
\hline$\hat{\mathbb{I}}$ & $|1\rangle \hat{\mathbb{I}}\langle 2|, \lambda_{\mathbb{I}}^{12}$ & $|1\rangle \hat{\mathbb{I}}\langle 3|, \lambda_{\mathbb{I}}^{13}$ & $|2\rangle \hat{\mathbb{I}}\langle 3|, \lambda_{\mathbb{I}}^{23}$ \\
\hline
\end{tabular}

The basis of coherent vibrational states allows us to simplify the computation of the Lagrange multipliers in terms of the time-dependent matrix elements of the exact surprisal. In what follows we assume that Eq. (11) is an accurate numerical approximation at all the times of interest for the dynamics. The matrix elements of the surprisal then are given 
as a sum of the matrix elements of the time-independent constraints listed in Table I with their time-dependent coefficients, the Lagrange multipliers. As we discussed, these matrix elements of the constraints are evaluated in an initial, time-independent basis of the coherent states, Eq. (13).

The only operator that contributes to the $\langle 0, i|\hat{I}| j, 0\rangle(t)$ matrix element of the surprisal for the coherent state basis function with $\langle\hat{R}\rangle_{\alpha_{k}}=0$ and $\left|\alpha_{k}\right|=0$ is the identity operator, $|i\rangle \hat{\mathbb{I}}\langle j|$. This diagonal in the vibrational index matrix element for the identity operator, $\langle 0, i|\hat{\mathbb{I}}| j, 0\rangle$, equals to 1 , Eq. (9). Thereby one can identify the Lagrange multipliers for the diagonal and off-diagonal in the electronic index operators, $|i\rangle \hat{\mathbb{I}}\langle j|$ as:

$$
\lambda_{\mathbb{I}}^{i j}(t)=\langle 0, i|\hat{I}| j, 0\rangle(t)
$$

Assuming that the surprisal can be described by the set of constraints listed in Table I, we also need to identify the Lagrange multipliers for the operators $\hat{a}^{\dagger} \hat{a}$ of the different electronic states, see TableI Ia. Considering a diagonal matrix element of the surprisal, $\left\langle\alpha_{k}, i|\hat{I}| i, \alpha_{k}\right\rangle(t)$, if $\left|\alpha_{k}\right| \neq 0$ the only two operators that contribute to it are $|i\rangle \hat{\mathbb{I}}\langle i|$ and $|i\rangle \hat{a}^{\dagger} \hat{a}\langle i|$.

$$
\left\langle\alpha_{k}, i|\hat{I}| i, \alpha_{k}\right\rangle(t)=\lambda_{\mathbb{I}}^{i i}(t)\left\langle\alpha_{k}, i|\hat{\mathbb{I}}| i, \alpha_{k}\right\rangle+\lambda_{a^{\dagger} a}^{i i}(t)\left\langle\alpha_{k}, i\left|\hat{a}^{\dagger} \hat{a}\right| i, \alpha_{k}\right\rangle
$$

According to Eq. (9) the first constraint contributes only with $\lambda_{\mathbb{I}}^{i i}(t)$, and the $\left\langle\alpha_{k}, i\left|\hat{a}^{\dagger} \hat{a}\right| i, \alpha_{k}\right\rangle$ matrix element equals to $\left|\alpha_{k}\right|^{2}$. The Lagrange multipliers for this constraint are then computed as follows:

$$
\lambda_{a^{\dagger} a}^{i i}(t)=\frac{1}{\left|\alpha_{k}(0)\right|^{2}}\left(\left\langle\alpha_{k}, i|\hat{I}| i, \alpha_{k}\right\rangle(t)-\lambda_{\mathbb{I}}^{i i}(t)\right)
$$

where $\alpha_{k}$ can be any of the basis functions except the one with $\langle\hat{R}\rangle_{\alpha_{k}}=0$. We verify the accuracy of the approximation Eq. (11) and computed Lagrange multipliers by comparing the dynamics of both populations and coherences with those obtained from the wave function propagation on a grid. The transformation to the density of the approximate surprisal is similar to the exact one, given by Eq. (7), as discussed in section II.B.

\section{RESULTS AND DISCUSSION}

There is a time gap following the excitation until the subsequent mass dependent transfer between the excited diabatic states. It is the duration required for the excited wave packet 
on the shifted potential to complete a vibrational cycle and return to the Franck Condon region. We deal first with the earlier time scale and demonstrate that the dominant constraints reproduce the dynamics. Then we show that the same set stays relevant also during the transfer. The key conclusion is that the oscillatory change in time of the coherence governs the rate of transfer and that the mass dependence is fully captured by the density as determined by the constraints.

\section{A. Dominant constraints during an atto-second excitation}

The set of constraints needed to describe the initial state, the ground vibrational state of the ground electronic state, consists of two operators in the nuclear Hilbert subspace and three operators in electronic subspace: $\left\{\hat{\mathbb{I}}, \hat{a}^{\dagger} \hat{a}\right\} \otimes\{|1\rangle\langle 1|| 2\rangle,\langle 2|| 3\rangle,\langle 3|\}$, see section II.B. Results for the dynamics of this initial state during an ultrafast excitation are shown on Fig. 2 as computed from the wave function propagated on a grid compared with the picture given by the set of 9 dominant constraints, Table [. Interaction with the laser pulse induces population transfer to the excited electronic states in the Franck-Condon region and gives rise to the off-diagonal elements of the density that are coherences between different electronic states. The onset of the coherence is reflected by the rapid rise of the Lagrange multipliers of the identity operator that is off-diagonal in the electronic index, $|i\rangle \hat{\mathbb{I}}\langle j|$ (Table Ib), see Fig. 3.

The values of the multipliers for the operators $|i\rangle \hat{\mathbb{I}}\langle i|$, diagonal in the electronic index, are also changing during the excitation, as shown in Fig. 3a. The rise in $\lambda_{\mathbb{I}}^{11}$ of the ground electronic state goes together with the decrease in the excited state multipliers $\lambda_{\mathbb{I}}^{22}$ and $\lambda_{\mathbb{I}}^{33}$, preserving the value of the sum: $\lambda_{\mathbb{I}}^{11}+\lambda_{\mathbb{I}}^{22}+\lambda_{\mathbb{I}}^{33}$. This is also true for the Lagrange multipliers of the $|i\rangle \hat{a}^{\dagger} \hat{a}\langle i|$ operator, although the latter do not change significantly during the dynamics. The diagonal in the electronic index operators are the only ones which contribute to the trace of the surprisal. These relations between the Lagrange multipliers follow from the trace being unaltered under a unitary transformation: $\operatorname{Tr}(\ln \hat{\rho}(t))=\operatorname{Tr}\left(\hat{U} \ln \hat{\rho}(0) \hat{U}^{\dagger}\right)=\operatorname{Tr}(\ln \rho(0))$.

The ratio of the values $\lambda_{\mathbb{I}}^{22}$ and $\lambda_{\mathbb{I}}^{33}(\approx 1 / 7)$ is similar to the ratio of the populations in these states after the pulse, which is governed by the ratio of the square of the transition dipole moments. As seen in Fig. 3a after the field is over the Lagrange multipliers for the operators diagonal in the electronic index do not change with time. At the same time-range the oscillatory dynamics of the off-diagonal Lagrange multipliers $\left\{\lambda_{\mathbb{I}}^{12}, \lambda_{\mathbb{I}}^{13}, \lambda_{\mathbb{I}}^{23}\right\}$ is governed 
(a)

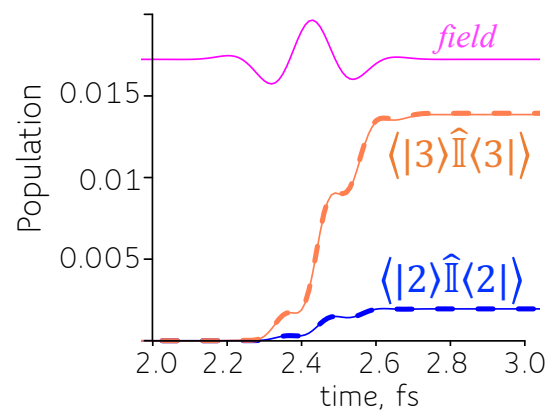

(b)

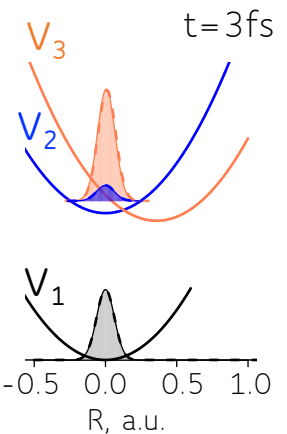

FIG. 2. Mean values and population profiles computed from the wave function propagation on the grid (solid lines) and via dominant set of 9 constraints (dashed lines), see Table I (a) Population dynamics in excited electronic states $\langle\mid 2\rangle \hat{\mathbb{I}}\langle 2 \mid\rangle$ and $\langle\mid 3\rangle \hat{\mathbb{I}}\langle 3 \mid\rangle$ vs. time during the interaction with the laser pulse (time profile shown in pink). (b) Population distribution as a function of the nuclear coordinate on ground $\left(V_{1}\right.$, shown in black) and two excited ( $V_{2}, V_{3}$ shown in blue and orange color, respectively) electronic states just after the laser pulse is over, at about $t=3 \mathrm{fs}$.

(a)

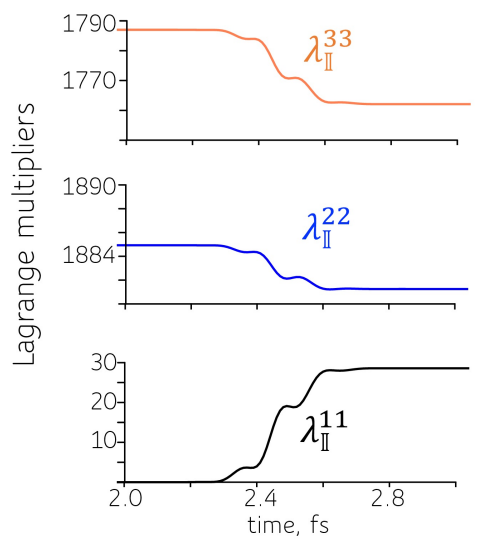

(b)

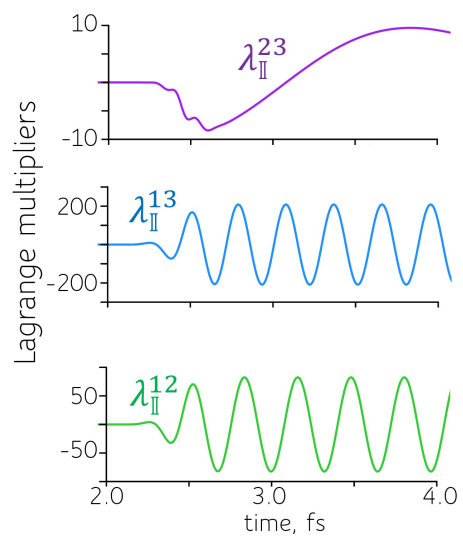

FIG. 3. Time-evolution of the Lagrange multipliers $\left(\lambda_{\mathbb{I}}^{i j}\right.$ in ${ }^{14} \mathrm{~N}_{2}$, see Table 1$)$ of the electronic states during the interaction with the pulse for operators (a) diagonal and (b) off-diagonal in the electronic index. The Lagrange multipliers of the operators diagonal in the electronic index are real. For the off-diagonal $\lambda_{\mathbb{I}}^{i j}$ and its complex conjugate, only the dynamics of the real part is shown. $\hat{\mathbb{I}}$ is the identity element in the space of vibrations.

by the energy difference of the potential energy of the states $V_{i}^{(0)}=V_{i}\left(R_{e q}\right)$, each taken at its equilibrium distance, see Table S1 in section S1 of the supplementary material. The oscillatory trends are very evident in the analytical expressions for the time dependence of 
the Lagrange multipliers for times after the field is over, section IV, Eq. (19).

\section{B. Isotope effect during the non-adiabatic transfer after interaction with the laser field}

Already during the interaction with the laser field, at $\approx 2.4 \mathrm{fs}$, the wave packet on the shifted potential begins to move out from the Franck-Condon region. Fig. 4 contrasts dynamics for the physically correct $\mathrm{N}_{2}$ with atomic mass of nitrogen 14 and an insilico mass of 10 . Heavier nuclei move slower: just after the pulse there is no significant difference in the population distribution between the two isotopomers, however the first vibrational cycle for mass 10 is completed almost 2 fs sooner compare to mass 14 (Fig. 4c).
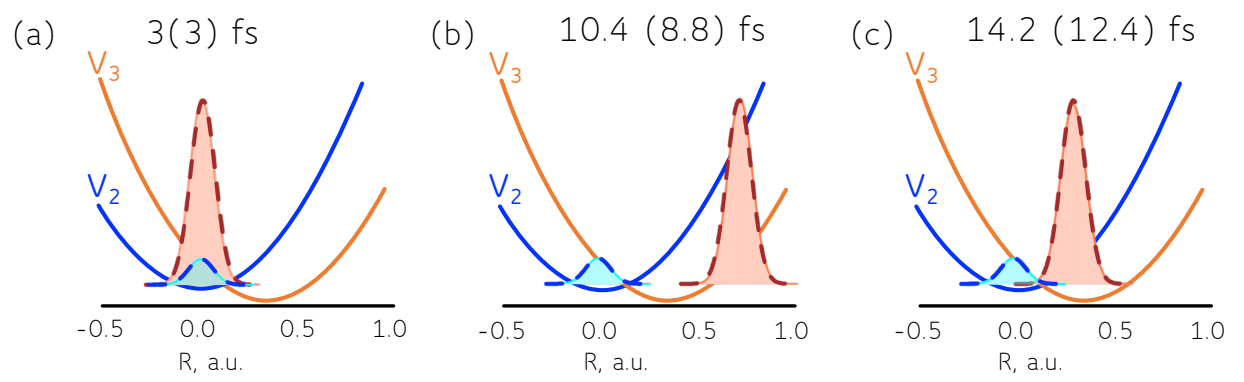

FIG. 4. Population dynamics for $\mathrm{N}_{2}$ at three different times as shown, for atomic mass 14. For the lighter mass 10 the time is given in parenthesis. The population distribution is presented for two excited $\left(\mathrm{V}_{2}, \mathrm{~V}_{3}\right.$ shown in blue and orange, respectively) electronic states as the dynamics unfolds: (a) just after the pulse; (b) when the $V_{3}$ wave packet reaches the outer turning point; (c) when the $V_{3}$ wave packet is entering the coupling region at the recurrence. Shown are two computations for the wave packets, a propagation on a grid, shaded area, and surprisal given by the dominant constraints (Table I), envelope in dashed lines.

This Newtonian effect of mass is reflected also in the coherence revival times, seen both in $\rho_{31}=\langle\mid 3\rangle \hat{\mathbb{I}}\langle 1 \mid\rangle$ and $\rho_{32}=\langle\mid 3\rangle \hat{\mathbb{I}}\langle 2 \mid\rangle$ dynamics (Fig. 5). The figure shows both exact numerical results for the dynamics as well as computations of the surprisal using the realistic set of nine constraints, see Table I. In such a procedure the time dependent Lagrange multipliers for the selected constraints are computed from the exact surprisal as discussed in section II.C. Using these Lagrange multipliers we get an accurate but approximate surprisal as in Eq. (11).

As the wave packet on the third electronic state goes out of the Franck-Condon region its overlap with the other two wave packets, and consequently their coherence, goes to zero. 
In addition to this essentially classical isotope effect there is also a noticeable change in the phase of the slow $\rho_{32}$ coherence, Fig. 5b. It is this coherence that governs the rate and the direction of non-adiabatic transfer between the two excited states, as shown in Fig. 6. If for mass 10 the sign of the first strong peak in the coherence is negative, which corresponds to the loss of population from the shifted electronic state, for mass 14 the first peak is positive, and as a result, the direction of the initial transfer is reversed. Both classical and quantum
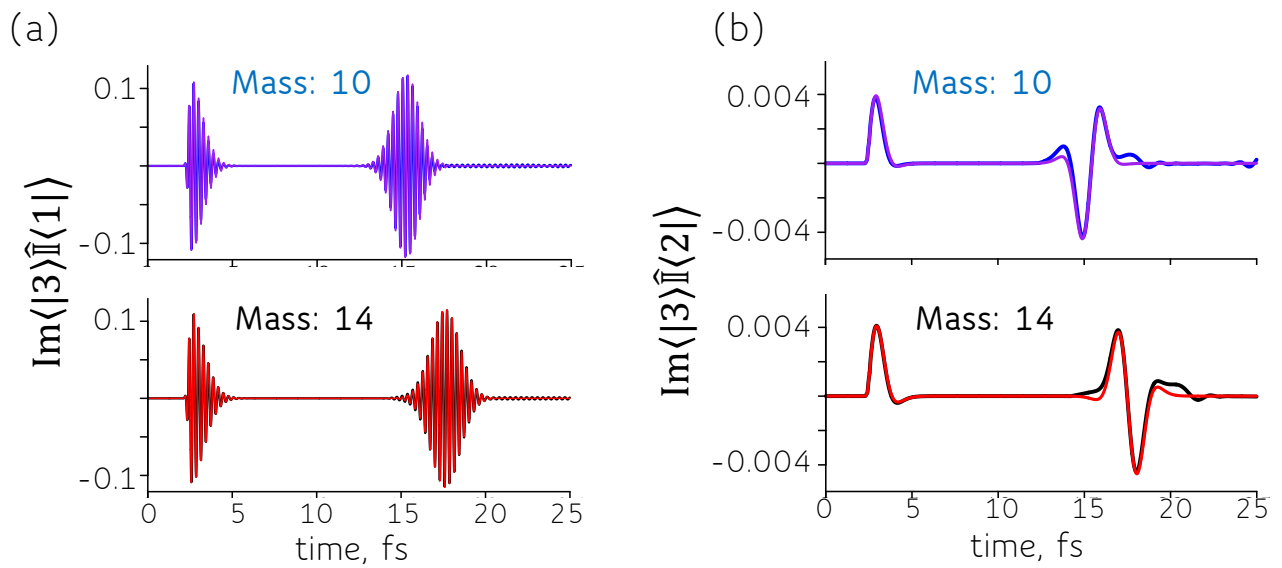

FIG. 5. Effect of the mass on the revival of two coherences $\operatorname{Im}\langle\mid 3\rangle \hat{\mathbb{I}}\langle 1 \mid\rangle$ (a) and $\operatorname{Im}\langle\mid 3\rangle \hat{\mathbb{I}}\langle 2 \mid\rangle$ (b) for $N_{2}$ with the atomic mass 14 and the mass 10 (bottom and top panels, respectively). Darker solid lines (black and blue) represent results for the wave function propagation on a grid, brighter colors (red and purple) are results for the computation of the surprisal given by the dominant set of constraints, Table $\mathrm{I}$

isotope effects in the dynamics of the coherences are well reproduced by the computation via the dominant set of constraints. The largest error is in the tails of the coherences because the wave function computed on the grid is a shade broader than a Gaussian.

The number of dominant constraints that are needed to accurately capture both the Newtonian and the quantum effect of mass stays the same as during the interaction with the laser field (Table $\mathbb{I}$ ). The Lagrange multipliers $\lambda_{\mathbb{I}}^{23}$ and $\lambda_{\mathbb{I}}^{13}$ are most strongly affected by the mass starting to be different already around 4 fs, see Fig. 7, where there is not yet coupling of the diabatic states. In order to understand this non-trivial effect of mass on the Lagrange multipliers we consider the analytical form of the time-dependent surprisal for multiple electronic states in the absence of coupling of diabatic states in the time-evolution operator. 


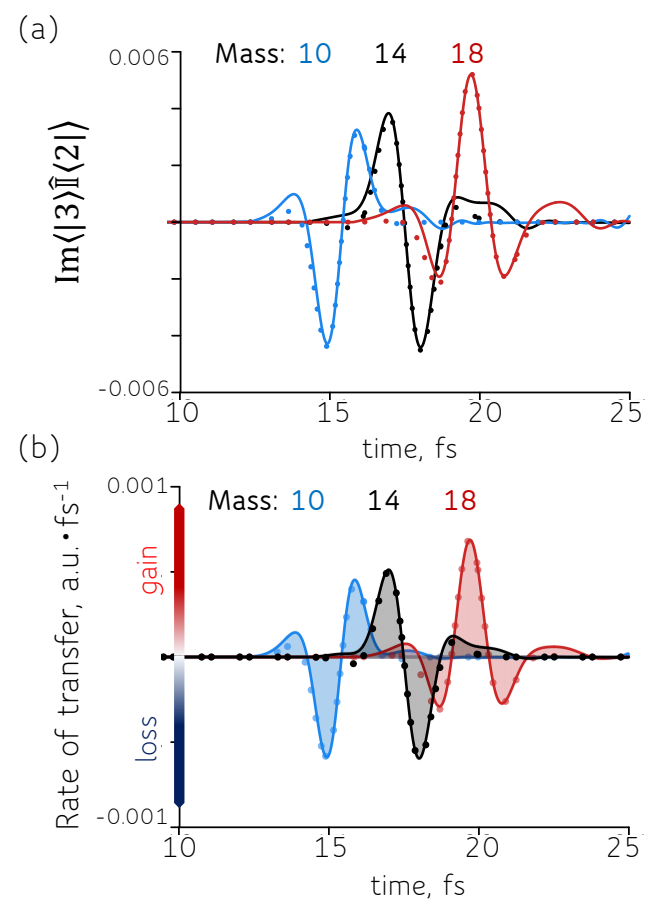

FIG. 6. Effect of the mass on the coherence $\operatorname{Im}\langle\mid 3\rangle \hat{\mathbb{I}}\langle 2 \mid\rangle$ (a) and the rate of transfer in the shifted electronic state (b) between the two excited electronic states in the coupling region for atomic masses of nitrogen: 10 (blue), 14 (black) and 18 (red). One-to-one correspondence between the two panels is evident. Shown are two sets of computational results: a wave function propagation on a grid (solid lines) and the surprisal as represented by the dominant set of constraints (dots), see Table I.

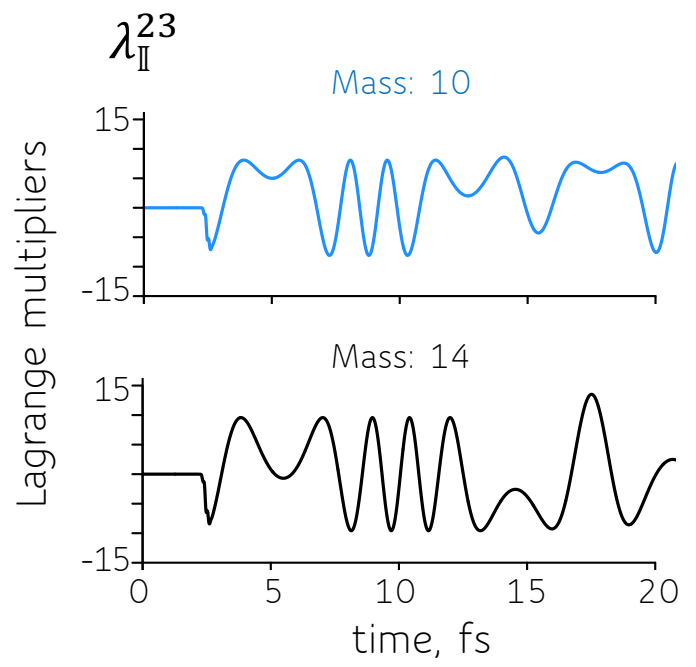

FIG. 7. The real part of the Lagrange multiplier $\lambda_{\mathbb{I}}^{23}$ of the constraint $|2\rangle \hat{\mathbb{I}}\langle 3|$ for two masses of nitrogen atom: 10 (top panel) and 14 (bottom panel). The non-linear time-dependent effect of mass is clear. The computation uses Eqs. 1416 . 


\section{DYNAMICS OF THE LAGRANGE MULTIPLIERS: ANA- LYTICAL FORM OF THE TIME-DEPENDENT SURPRISAL AND THE EFFECT OF MASS}

After the laser field is over, in the absence of diabatic coupling terms the time-evolution operator is diagonal in the electronic indices. It can be written as:

$$
\hat{U}=\exp \{-i \hat{H} t\}=\sum_{i}|i\rangle \exp \left\{-i \hat{h}_{i} t\right\}\langle i|
$$

where $\hat{H}$ is the sum of only the harmonic Hamiltonians $\hat{h}_{i}$ of the three uncoupled electronic states, namely the first three terms of Eq. (1). The dynamics under this separable form simplify and allows an analytical derivation of the time-dependent surprisal, see detailed derivation in section S1 of the supplementary material. Those matrix elements diagonal in the electronic index appear to be not changing with time:

$$
\begin{aligned}
& \left\langle\alpha_{k}\left|\hat{I}^{11}(t)\right| \alpha_{m}\right\rangle=\lambda_{\mathbb{I}}^{11}\left(t_{s}\right)\left\langle\alpha_{k}|\hat{\mathbb{I}}| \alpha_{m}\right\rangle+\lambda_{a^{\dagger} a}^{11}\left(t_{s}\right)\left\langle\alpha_{k}\left|\hat{a}^{\dagger} \hat{a}\right| \alpha_{m}\right\rangle \\
& \left\langle\alpha_{k}\left|\hat{I}^{22}(t)\right| \alpha_{m}\right\rangle=\lambda_{\mathbb{I}}^{22}\left(t_{s}\right)\left\langle\alpha_{k}|\hat{\mathbb{I}}| \alpha_{m}\right\rangle+\lambda_{a^{\dagger} a}^{22}\left(t_{s}\right)\left\langle\alpha_{k}\left|\hat{a}^{\dagger} \hat{a}\right| \alpha_{m}\right\rangle \\
& \left\langle\alpha_{k}(t)\left|\hat{I}^{33}(t)\right| \alpha_{m}(t)\right\rangle=\lambda_{\mathbb{I}}^{33}\left(t_{s}\right)\left\langle\alpha_{k}|\hat{\mathbb{I}}| \alpha_{m}\right\rangle+\lambda_{a^{\dagger} a}^{33}\left(t_{s}\right)\left\langle\alpha_{k}\left|\hat{a}^{\dagger} \hat{a}\right| \alpha_{m}\right\rangle
\end{aligned}
$$

here $\left|\alpha_{j}\right\rangle$ are the basis functions, vibrational coherent states placed on each electronic state. Note even so the basis functions $\left|\alpha_{j}(t)\right\rangle$ on the shifted electronic state $|3\rangle$ are time-dependent, the matrix elements of the constraints are taken in the initial non-shifted basis $\left|\alpha_{j}\right\rangle$, see discussion in section S1.5 of the supplementary material. The time $t_{s}$ is considered to be the time when the pulse is over, at about $3 \mathrm{fs}$ of the actual dynamics, Fig. 2 . The values of the Lagrange multipliers at this time point are taken from the exact surprisal propagation, as described in section II.C.

The off-diagonal in the electronic index matrix elements of the surprisal change with time only due to the Lagrange multipliers of the identity operator, Table \b:

$$
\begin{aligned}
& \left\langle\alpha_{k}\left|\hat{I}^{12}(t)\right| \alpha_{m}\right\rangle=\lambda_{\mathbb{I}}^{12}\left(t_{s}\right) e^{i \Omega_{12} \tau}\left\langle\alpha_{k}|\hat{\mathbb{I}}| \alpha_{m}\right\rangle=\lambda_{\mathbb{I}}^{12}(t)\left\langle\alpha_{k}|\hat{\mathbb{I}}| \alpha_{m}\right\rangle \\
& \left\langle\alpha_{k}\left|\hat{I}^{13}(t)\right| \alpha_{m}(t)\right\rangle=\lambda_{\mathbb{I}}^{13}\left(t_{s}\right) e^{i \Omega_{13} \tau} \Theta(\tau)\left\langle\alpha_{k}|\hat{\mathbb{I}}| \alpha_{m}\right\rangle=\lambda_{\mathbb{I}}^{13}(t)\left\langle\alpha_{k}|\hat{\mathbb{I}}| \alpha_{m}\right\rangle \\
& \left\langle\alpha_{k}\left|\hat{I}^{23}(t)\right| \alpha_{m}(t)\right\rangle=\lambda_{\mathbb{I}}^{23}\left(t_{s}\right) e^{i \Omega_{23} \tau} \Theta(\tau)\left\langle\alpha_{k}|\hat{\mathbb{I}}| \alpha_{m}\right\rangle=\lambda_{\mathbb{I}}^{23}(t)\left\langle\alpha_{k}|\hat{\mathbb{I}}| \alpha_{m}\right\rangle
\end{aligned}
$$

here $\tau=t-t_{s}$ is a shifted time of the dynamics. The oscillation frequencies are given by:

$\Omega_{12}=V_{2}^{(0)}, \Omega_{13}=V_{3}^{(0)}+\kappa_{0}$ and $\Omega_{23}=V_{3}^{(0)}-V_{2}^{(0)}+\kappa_{0}$. The parameter $\kappa_{0}=(1 / 2) \cdot k R_{e q}^{2}$ 
is mass-independent and accounts for the change in the energy due to the shift of the $V_{3}$ potential to $R_{e q}$, see Table S1 in section S1 of the supplementary material for the parameters of the harmonic potentials.

The only mass-dependent factor in Eq. (19) is the complex valued $\Theta(\tau)$. Its phase is determined by the shift of the upper potential. Its origin is the non commutativity of the shift and the number operators of the harmonic potential in the time evolution operator for motion on this potential, see Eqs. (S1.13-S1.15) in section S1 of the supplementary material. The very same phase factor appears also for a more general initial states when a larger set of dominant constraints is needed, section S1.6 of the supplementary material. Such a generalization is useful for example when the interaction with the field is through an R-dependent transition dipole.

$\Theta(\tau)$ contributes to the matrix elements of $\hat{I}^{13}(t)$ and $\hat{I}^{23}(t)$, with $\xi=\sqrt{m \omega / 2} \cdot R_{e q}$ being proportional to the shift in the equilibrium position $R_{e q}$ of the $V_{3}$ potential. The effect is quadratic in the dimensionless parameter $\xi$ :

$$
\Theta(\tau)=\exp \left\{-i \xi^{2}(\omega \tau-\sin (\omega \tau))\right\}
$$

The analytical form of the surprisal matrix elements given by Eq. 18,19 fully captures the effect of mass, both classical and quantum, see Fig. S1 in section S4 of the supplementary material. The revival of the low-frequency coherence $\operatorname{Im}\langle\mid 3\rangle \hat{\mathbb{I}}\langle 2 \mid\rangle$ between the two excited states occurs at later times for heavier masses, and the oscillation of the coherence and its change with the mass is reproduced on a quantitative level as compared to the numerical surprisal represented by the dominant set of constraints.

The role of the mass-dependent factor $\Theta(\tau)$ in the Lagrange multipliers $\lambda_{\mathbb{I}}^{13}(t)$ and $\lambda_{\mathbb{I}}^{23}(t)$, Eq. (19), on the overall dynamics can be seen explicitly by contrasting the results with those computed with $\Theta(\tau)=1$, see Fig. 8. Without this factor only the classical effect of mass is accurately captured (Fig. 8b), which is described by the time-dependent shift operator in the vibrational coherent basis set, Eq. (4). There is no change in the interference pattern upon the change in atomic mass. The mass-dependence of the $\Theta(\tau)$ factor originates in $\xi$. This phase-factor is crucially needed to reproduce the quantum effect of the mass on the electronic coherence.

The highly non-linear effect of the mass on the dynamics of the Lagrange multipliers is 
(a)

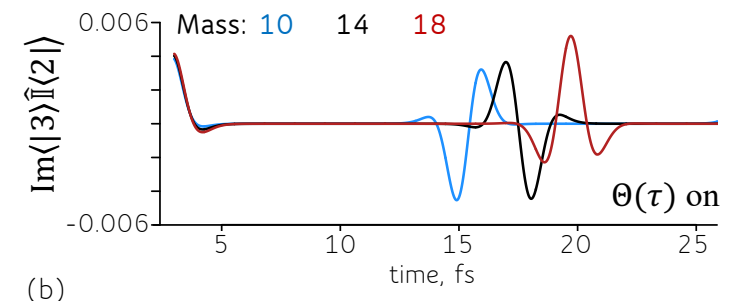

(b)

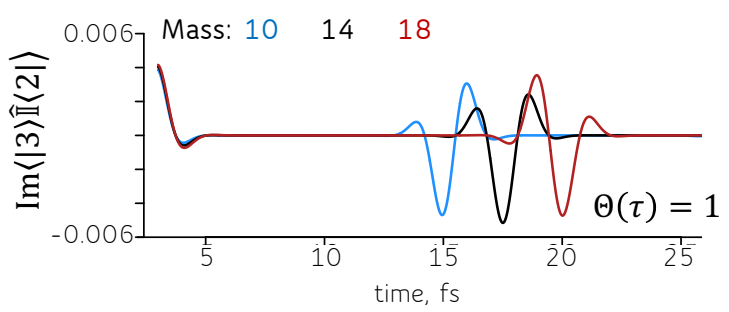

FIG. 8. Coherence between the two excited states $\operatorname{Im}\langle\mid 3\rangle \hat{\mathbb{I}}\langle 2 \mid\rangle$ computed using analytical form of the time-dependent surprisal, Eqs. 18 19): (a) with the non-zero mass-dependent phase factor $\xi$ in the $\Theta(\tau)$ governed by the shift in equilibrium distance of the $V_{3}$ potential; (b) with $\Theta(\tau)=1$ throughout the dynamics. Without the mass-dependent phase-factor analytical results reproduce only the Newtonian effect of mass. The essentially quantum isotope effect in the interference is due to the non-zero $\xi$ phase-factor in the $\Theta(\tau)$.

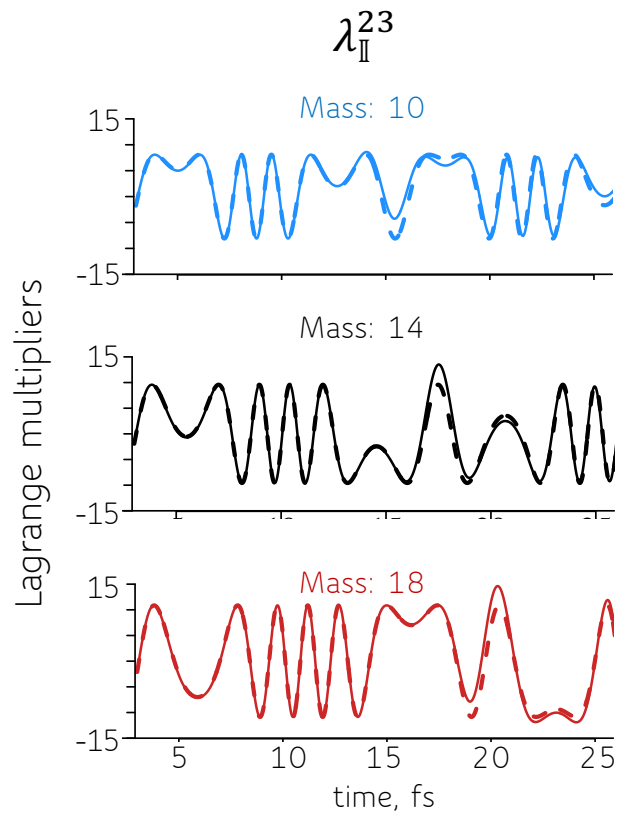

FIG. 9. The real part of the Lagrange multiplier $\lambda_{\mathbb{I}}^{23}$ for the coherence operator $|2\rangle \hat{\mathbb{I}}\langle 3|$ for different masses computed from the exact surprisal using Eqs. $\sqrt{14} 16$ (solid lines) and via Eq. 19 (dashed lines). 
also fully reproduced by the Eq. (19), see for example Fig. 9 for $\lambda_{\mathbb{I}}^{23}(t)$. The same Lagrange multiplier but computed taking $\Theta(\tau)=1$ shows only oscillatory behavior, governed by the $e^{i \Omega_{23} \tau}$ phase-factor, Fig. S2 in section $\mathrm{S} 4$ of the supplementary material. In the time-evolution of the $\lambda_{\mathbb{I}}^{23}(t)$ there are two regimes: (i) oscillatory dynamics, 8-14 fs for mass 14 for example; (ii) more stationary dynamics, 14-17 fs for mass 14. Similar features can be seen for other masses, but for different time-windows. These time-windows correlate with the time ranges of the effective transfer of the population, Fig. 6 .

The switch between the two regimes is governed by the dynamics of the phase-factor in the $\Theta(\tau)$, see Fig. 10. The amplitude transfer is effective during the regime of stationary phase. The direction of the transfer is determined by the value of the stationary phase. Fig. 10 is a summary that highlights the role of the mass in the coupling between the two states.

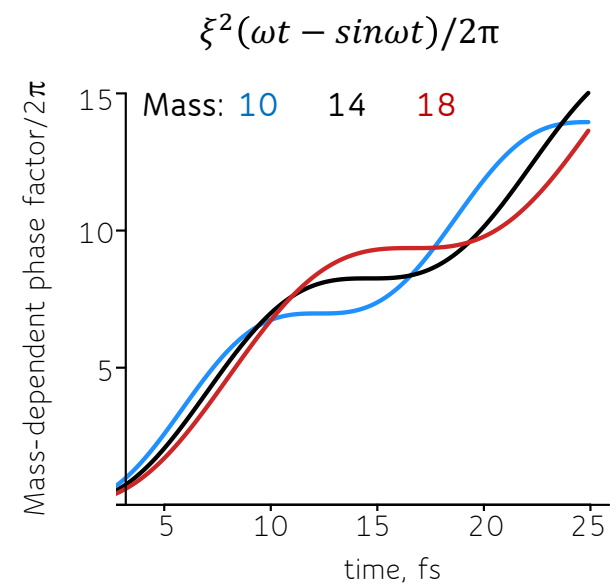

FIG. 10. Rapid growth of the mass-dependent phase factor $\xi^{2}(\omega t-\sin \omega t) / 2 \pi$ as the dynamics unfolds over the region of curve crossing. There is evident a stationary phase region where the vibrational wave packets on the two electronic states interfere constructively.

The phase-factor of $\Theta(\tau)$ is rapidly changing with time in the interval when the wave packet on state 3 is moving to the outer turning point and back towards the Franck Condon region (Fig. 4). A heavier mass takes a little longer to cover this path. The rapid growth of the phase with time leads to different phases when the different isotopomers reach the coupling region. This affects the direction of transfer between the states, as seen in Fig. 6. 


\section{CONCLUSIONS}

We presented an algebraic based dynamical surprisal analysis for an electronically attoexcited diatomic molecule. The excited states interact by non-adiabatic coupling that we rewrote as coupling of diabatic states by a potential. In the absence of this potential we can identify an algebra of electronic-vibrational operators with few members, an algebra that accounts exactly for the dynamics following the excitation of a thermal initial state. This set of nine operators, given in Table I is sufficient to provide a close approximation for the surprisal even when the states are coupled, see Fig. 6. Specifically, three of the nine operators are non diagonal in the electronic state index, $|1\rangle \hat{\mathbb{I}}\langle 2|| 1,\rangle \hat{\mathbb{I}}\langle 3|$ and $|2\rangle \hat{\mathbb{I}}\langle 3|$.

The analytic expressions for the time dependence of the Lagrange multipliers allows a compact representation of the dynamics of the surprisal. The analytic matrix elements of the surprisal in the absence of coupling of the diabatic states are written more simply by working in a time-dependent basis of coherent states. On the shifted potential the Franck Condon region is displaced with respect to the equilibrium distance. Therefore the initial vibrational states excited on this potential are non stationary. It is therefore advantageous to use time-dependent displaced coherent states.

The surprisal is expanded as an exact sum of the elements of the closed algebra, each multiplied by its conjugate time-dependent Lagrange multiplier. This allows an explicit analytic description of the role of the mass in the dynamics. The quantum mechanical isotope effect is quantitatively captured in an analytic expression, see Fig. 8, by a complex number of unit modulus in the Lagrange multiplier of the coherence operator between the excited states, Eq. (19). The phase of this complex number represents the interference between the wave packets moving on the two different electronic states. Explicitly this phase, $\xi^{2}(\omega t-\sin \omega t)$, is a product of $\xi$, a dimensionless variable proportional to the displacement of the upper electronic state and a time factor. Both factors depend in a known analytic way on the mass. When this phase is stationary, Fig. 10, the transfer between the two states is effective. The direction of the transfer, gain or loss, is mass dependent and is determined by the value of the phase at its stationary time interval. 


\section{SUPPLEMENTARY MATERIAL}

The supplementary material contains the analytical derivation of the time-dependent surprisal discussed in Sec. IV. It is followed by the computational details of the numerical propagation of the surprisal in a finite basis, in particular, details about the time-derivatives of the basis functions are specified. The numerical propagation of the wave function in a grid representation is discussed in Sec. S3. The final Sec. S4 shows additional figures supporting the results of the analytical derivation.

\section{ACKNOWLEDGEMENTS}

This work was supported by the EC Horizon2020 FETOpen COPAC Project No. 766563 and by the US-Israel grant 2019722 NSF-BSF Astronomy and Astrophysics. F.R. acknowledges support from the Fonds National de la Recherche Scientifique, Belgium (F.R.S-FNRS), research grant No. T.0132.16.

\section{DATA AVAILABILITY}

The data that support the findings of this study are within the article and its supplementary material.

\section{REFERENCES}

${ }^{1}$ A. H. Zewail and R. B. Bernstein, "Real-time laser femtochemistry viewing the transition from reagents to products", Chem. Eng. News 66, 24-43 (1988).

${ }^{2}$ F. Remacle and R. D. Levine, "An electronic time scale for chemistry", Proc. Natl. Acad. Sci. USA 103, 6793-6798 (2006).

${ }^{3}$ H. Nakamura, Nonadiabatic transition (World Scientific, Singapore, 2002).

${ }^{4}$ E. E. Nikitin and S. Umanskii, Theory of slow atomic collisions (Springer-Verlag Berlin Heidelberg, 1984).

${ }^{5}$ C. Zener, "Non-adiabatic crossing of energy levels", Proc. R. Soc. A 137, 696-702 (1932).

${ }^{6}$ J. S. Ajay, K. G. Komarova, F. Remacle, and R. D. Levine, "Time-dependent view of an isotope effect in electron-nuclear nonequilibrium dynamics with applications to n2", Proc. Natl. Acad. Sci. USA 115, 5890-5895 (2018) 
${ }^{7}$ M. Ben-Nun and R. D. Levine, "Short-time dynamics on several electronic states: formalism and computational study of i2 in rare gas solvents", Chem. Phys. 201, 163-187 (1995)

${ }^{8}$ E. J. Heller, "Time-dependent approach to semiclassical dynamics", J. Chem. Phys. 62, 1544-1555 (1975)

${ }^{9}$ Y. Alhassid and R. D. Levine, "Entropy and chemical change. iii. the maximal entropy (subject to constraints) procedure as a dynamical theory", J. Chem. Phys. 67, 4321-4339 (1977)

${ }^{10}$ R. Levine, "Surprisal analysis using dressed variables", Chem. Phys. Lett. 207, 265-269 (1993).

${ }^{11}$ K. Komarova, F. Remacle, and R. D. Levine, "Surprisal of a quantum state: dynamics, compact representation, and coherence effects", J. Chem. Phys. 153, 214105 (2020).

${ }^{12}$ R. D. Levine, "Information theory approach to molecular reaction dynamics", Annu. Rev. Phys. Chem. 29, 59-92 (1978)

${ }^{13}$ R. D. Levine and R. B. Bernstein, "Energy disposal and energy consumption in elementary chemical reactions. information theoretic approach", Acc. Chem. Res. 7, 393-400 (1974).

${ }^{14}$ P. R. Halmos and L. J. Savage, "Application of the Radon-Nikodym Theorem to the Theory of Sufficient Statistics", Ann. Math. Stat. 20, 225-241 (1949).

${ }^{15} \mathrm{M}$. Tribus and R. Levine, The maximum entropy formalism (The MIT Press, Cambridge, Massachusetts, and London, England, 1979), pp. 1-118.

${ }^{16}$ E. T. Jaynes, "Information theory and statistical mechanics", Phys. Rev. 106, 620-630 (1957)

${ }^{17}$ S. Dagan and Y. Dothan, "Evaluation of an incompletely measured spin density matrix", Phys. Rev. D 26, 248-260 (1982).

${ }^{18}$ A. Katz, Principles of statistical mechanics : the information theory approach (Freeman, San Francisco, 1967).

${ }^{19}$ M. Faist and R. Levine, "On the product electronic state distribution in reactions of alkali dimers with halogen atoms", Chem. Phys. Lett. 47, 5-10 (1977).

${ }^{20}$ W. S. Struve, J. R. Krenos, D. L. McFadden, and D. R. Herschbach, "Molecular beam kinetics: angular distributions and chemiluminescence in reactions of alkali dimers with halogen atoms and molecules", J. Chem. Phys. 62, 404-419 (1975)

${ }^{21}$ J. R. Krenos and J. C. Tully, "Statistical partitioning of electronic energy: reactions of alkali dimers with halogen atoms", The Journal of Chemical Physics 62, 420-424 (1975)

${ }^{22}$ M. S. Child, "Franck-condon transitions in multi-curve crossing processes", Faraday Discuss. Chem. Soc. 55, 30-33 (1973)

${ }^{23}$ D. Spelsberg and W. Meyer, "Dipole-allowed excited states of n2: potential energy curves, vibrational analysis, and absorption intensities", J. Chem. Phys. 115, 6438-6449 (2001). 
${ }^{24}$ A. Ben-Shaul, R. D. Levine, and R. B. Bernstein, "Entropy and chemical change. ii. analysis of product energy distributions: temperature and entropy deficiency", J. Chem. Phys. 57, 5427-5447 (1972).

${ }^{25}$ R. D. Levine and R. B. Bernstein, Molecular reaction dynamics (Oxford University Press, New York, 1974).

${ }^{26}$ M. J. Berry, "Golden rule calculation of product vibronic population inversions in photodissociation and related reactions", Chem. Phys. Lett. 29, 329-336 (1974).

${ }^{27}$ R. Kubo and Y. Toyozawa, "Application of the Method of Generating Function to Radiative and NonRadiative Transitions of a Trapped Electron in a Crystal", Prog. Theor. Phys. 13, 160-182 (1955)

${ }^{28}$ C. Cohen-Tannoudji, Quantum mechanics, vol. 1 (Wiley, New York, 1977).

${ }^{29}$ R. Kosloff, "Time-dependent quantum-mechanical methods for molecular dynamics", J. Phys. Chem. 92, $2087-2100(1988)$

${ }^{30}$ J. S. Ajay, K. G. Komarova, S. Van Den Wildenberg, F. Remacle, and R. D. Levine, "Chapter 9 attophotochemistry: coherent electronic dynamics and nuclear motion", in Attosecond molecular dynamics (The Royal Society of Chemistry, 2018), pp. 308-347. 